\title{
Intravitreal Anti-Vascular Endothelial Growth Factor Therapy for Diabetic Macular Edema in Clinical Practice of Single Center: Three-Year Outcomes
}

\author{
Janejit Choovuthayakorn ${ }^{a}$ Phichayut Phinyob, c Apichat Tantraworasin ${ }^{c, d}$ \\ Paradee Kunavisarut $^{\mathrm{a}}$ Direk Patikulsila $^{\mathrm{a}}$ Voraporn Chaikitmongkol ${ }^{\mathrm{a}}$ \\ Nawat Watanachai ${ }^{a}$ Kessara Pathanapitoon ${ }^{a}$ \\ aDepartment of Ophthalmology, Faculty of Medicine, Chiang Mai University, Chiang Mai, Thailand; bepartment \\ of Family Medicine, Faculty of Medicine, Chiang Mai University, Chiang Mai, Thailand; 'Clinical Epidemiology and \\ Clinical Statistics Center, Faculty of Medicine, Chiang Mai University, Chiang Mai, Thailand; dDepartment of Surgery, \\ Faculty of Medicine, Chiang Mai University, Chiang Mai, Thailand
}

\section{Keywords}

Diabetic macular edema · Visual impairment · Anti-vascular endothelial growth factor

\begin{abstract}
Introduction: The objective of this study was to explore visual and anatomical outcomes in patients who underwent intravitreal anti-vascular endothelial growth factor (antiVEGF) injection for visual impairment from center-involved diabetic macular edema (Cl-DME) in clinical practice. Methods: Medical records of consecutive Cl-DME patients who initiated treatment with intravitreal bevacizumab injection between January 2012 and December 2016 and were followed for at least 12 months were retrospectively reviewed. Visual and anatomical changes after treatment over a 36-month period were evaluated. Results: There were 286 patients (423 eyes) with a mean (standard deviation, SD) age of 56.8 (8.5) years included in this study. One hundred and forty-six (51\%) patients were female, and 137 (47.9\%) patients received bilateral eye treatment. Mean (SD) present-
\end{abstract}

karger@karger.com www.karger.com/ore

Karger" ing visual acuity (VA) of overall eyes was 50.2 (19.6) letter scores. Stratified by baseline vision, eyes with initial VA worse than 20/40 achieved a statistically significant VA improvement, compared to baseline, by $+8.4,+6.9$, and +5.4 letters at 12, 24, and 36 months, respectively, with all $p$ values $<0.001$. However, when initial VA was $20 / 40$ and better, a non-statistically significant change in mean VA by +2.0 , -3.5 , and -3.6 letters were noted at the same time point ( $p$ value between 0.078 and 0.273 ). Unlike visual changes, a statistically significant decline in central subfield thickness compared to baseline was noted at the end of months 12 , 24 , and 36 in both initial VA subgroups (all $p$ values $<0.001$ ). Nevertheless, even though the median number of given injections considerably decreased from 6 in the first 12 months to 2 in the second 12-month period and 1 in the final 12-month interval, required ophthalmic clinic visits decreased in frequency with median numbers of 10, 7, and 6 appointments in each consecutive 12-month duration. Conclusion: This study supports the benefits of practical intravitreal anti-VEGF utilization to manage CI-DME in realworld settings. The improvement of vision in eyes present- 
ing with poor baseline $V A$ and maintenance of vision in eyes with better baseline VA were demonstrated through the 3-year review of each case. However, the burden of frequent monitoring warrants further evaluation of long-term compliance and efficacy.

(c) 2020 S. Karger AG, Basel

\section{Introduction}

Globally, as the incidence of diabetes mellitus (DM) increases, the vision-threatening conditions related to $\mathrm{DM}$, including diabetic retinopathy (DR) and diabetic macular edema (DME), are becoming major public health concerns $[1,2]$. A significant compromise in vision-related quality of life has been reported in diabetic patients who develop DR and/or DME, especially in ones with bilateral involvement $[3,4]$. For DME, to manage visual disability, different treatment modalities have been investigated [5-7]. In the past, macular laser photocoagulation was proposed as the effective treatment to reduce the risk of moderate visual loss among clinically significant macular edema patients [8]. However, with an evolution of intravitreal anti-vascular endothelial growth factor (antiVEGF) injection, considerable improvements in vision and central retinal thickness have been demonstrated to be superior to the previous macular laser photocoagulation in several standardized clinical trials [9-15]. At present, anti-VEGF injection has become a preferred initial treatment option for center-involved DME (CI-DME) in most clinical practices. Nevertheless, based on limited accessibility to health-care services, poor systemic conditions, and/or nonadherence to the treatment by patients, individualized treatment regimen varies substantially in clinical practice, mostly resulting in less frequent injections and less clinic visits than those recommended by strictly monitored clinical trials [16-19]. Then, the less favorable outcomes following anti-VEGF therapy for DME in clinical practice have been a concern [20-24]. Therefore, assessing the long-term benefit of intravitreal anti-VEGF therapy for DME in real clinical practice may add more valuable information for treatment decisions.

This study aimed to evaluate visual and anatomical outcomes following intravitreal anti-VEGF injection in DME patients who completed at least 12 months of follow-up in a tertiary referral center in Northern Thailand. The information of vision response would provide supportive evidence for DME patients to refine visual expectations, perceive the benefits and barriers, and increase their compliance once initiating anti-VEGF therapy.

\section{Materials and Methods}

This retrospective observational study was conducted in compliance with the tenets of the Declaration of Helsinki. The study protocol was reviewed and approved by the Research Ethics Committee, Faculty of Medicine, Chiang Mai University. The medical records of consecutive diabetes patients who were diagnosed as CI-DME with visual impairment, either treatment-naive or postmacular laser photocoagulation of $>3$ months before inclusion, and had initiated the first intravitreal anti-VEGF injection between January 2012 and December 2016 were identified. There was no direct patient or public involvement in this study. Informed consent was waived due to anonymized data extraction without compromising the privacy of the subjects. To be included in this study, the patient had to fulfill the following inclusion criteria: (1) had at least 1 anti-VEGF injection, (2) aged $\geq 18$ years, (3) had baseline macular central subfield thickness (CSFT), 1,000 $\mu$ m diameter circle centered at the fovea, $>320 \mu \mathrm{m}$ in males and $305 \mu \mathrm{m}$ in $\mathrm{fe}$ males measured by spectral domain optical coherence tomography (OCT) [25], (4) had initial visual acuity (VA) of 20/32 or worse, and (5) had a medical record of follow-up for at least 12 months, including the initial visit within 30 days after the first injection. Patients who had secondary macular edema from other retinal diseases, not DME, concomitant ocular diseases that would impact interpretation of macular thickness and VA, a history of macular or retinal disorders, a history of vitreoretinal surgery, had undergone cataract surgery or macular laser photocoagulation within 3 months prior to anti-VEGF injection, and received previous DME treatment by intravitreal anti-VEGF and/or steroid injection were excluded. For patients with bilateral anti-VEGF treatment, both eyes were included. Baseline characteristics, including age, gender, duration of diabetes, and other systemic medical diseases, were reviewed and recorded on a standardized form. At baseline and each follow-up visit, ocular characteristics, including best corrected VA, anterior segment findings by slit-lamp examination, intraocular pressure, and posterior segment findings by $90 \mathrm{D}$ non-contact lens, were extracted.

\section{OCT Analysis}

All OCT scans were obtained by Spectralis HRA-OCT (Heidelberg Engineering, Heidelberg, Germany) at the first and follow-up visits. CSFT values automatically measured by incorporated machine software were recorded. A horizontal OCT B-scan passing through the fovea (i.e., an area within $500 \mu \mathrm{m}$ of the foveal center) was also reviewed for the presence of intraretinal cysts and subretinal fluid at baseline. Additionally, the presence of disorganization of retinal inner layer (DRIL) and discontinuation of external limiting membrane (ELM) of $50 \%$ or greater were also assessed.

\section{Intravitreal Anti-VEGF Therapy}

At our center, 3 initial monthly loading injections were usually recommended; however, this was at the physician's discretion. Retreatment was administered based on non-stability in VA and CSFT criteria (e.g., VA change of at least 1 Snellen line or a change in CSFT of at least 10\%). In cases of stability in VA or CSFT, the injection was withheld and the next monitoring visit was extended to bi-monthly and quarterly, respectively. Injections were resumed with worsening VA and/or CSFT. Additional macular laser photocoagulation was considered in patients with VA and CSFT stabilization but residual macular thickening. In cases of no response 
Table 1. Demographics and ocular characteristics at presentation of DME eyes stratified by baseline VA

\begin{tabular}{|c|c|c|c|c|}
\hline Demographics and ocular characteristics & $\begin{array}{l}\text { Overall } \\
N=423 \text { eyes }\end{array}$ & $\begin{array}{l}\text { VA worse than } 20 / 40 \\
\text { (moderate to severe } \\
\text { visual impairment) } \\
N=310 \text { eyes }\end{array}$ & $\begin{array}{l}\text { VA } 20 / 40 \text { and } \\
\text { better (mild visual } \\
\text { impairment) } \\
N=113 \text { eyes }\end{array}$ & $p$ value \\
\hline \multicolumn{5}{|l|}{ Baseline demographic characteristics } \\
\hline Mean HbA1c level (SD), \% $(n=108)$ & $8.6(2.3)$ & $8.6(2.2)$ & $8.8(2.6)$ & 0.674 \\
\hline Median DM duration (IQR), year $(n=237)$ & $7(2-10)$ & $7(2-10)$ & $10(2.5-10)$ & 0.746 \\
\hline Median DME duration (IQR), month $(n=281)$ & $3(1-6)$ & $3(1-7)$ & $2(1-5.5)$ & 0.049 \\
\hline \multicolumn{5}{|l|}{ DR stage, $n(\%)$} \\
\hline Mild to moderate NPDR & $114(26.9)$ & $71(22.9)$ & $43(38.1)$ & \\
\hline Severe NPDR & $138(32.6)$ & $105(33.9)$ & $33(29.2)$ & 0.019 \\
\hline PDR & $171(40.4)$ & $134(43.2)$ & $37(32.8)$ & \\
\hline Previous macular laser photocoagulation, $n(\%)$ & $78(18.4)$ & $57(18.4)$ & $21(18.6)$ & 0.990 \\
\hline Previous PRP, $n(\%)$ & $147(34.8)$ & $109(35.2)$ & $38(33.6)$ & 0.818 \\
\hline Pseudophakia, $n$ (\%) & $50(11.8)$ & $39(12.6)$ & $11(9.7)$ & 0.498 \\
\hline \multicolumn{5}{|l|}{ Baseline OCT characteristics } \\
\hline CSFT, $\mu \mathrm{m}$, mean $(\mathrm{SD})$ & $496.8(160.3)$ & $525.3(169.8)$ & $418.7(94.2)$ & $<0.001$ \\
\hline Intraretinal cyst, $n(\%)$ & $336(79.4)$ & $253(81.6)$ & $83(73.5)$ & 0.077 \\
\hline DRIL, $n(\%)$ & $251(59.3)$ & $199(64.2)$ & $52(46.0)$ & $<0.001$ \\
\hline Subretinal fluid, $n$ (\%) & $208(49.2)$ & $154(49.7)$ & $54(47.8)$ & 0.743 \\
\hline $\mathrm{ERM}, n(\%)$ & $55(13.0)$ & $50(16.1)$ & $5(4.4)$ & $<0.001$ \\
\hline Disruption of ELM, $n(\%)$ & $127(30)$ & $119(38.4)$ & $8(7.1)$ & $<0.001$ \\
\hline
\end{tabular}

VA, visual acuity; HbA1c, serum glycated hemoglobin level; DM, diabetes mellitus; DME, diabetic macular edema; SD, standard deviation; IQR, interquartile range; DR, diabetic retinopathy; NPDR, non-proliferative diabetic retinopathy; PDR, proliferative diabetic retinopathy; PRP, pan-retinal photocoagulation; OCT, optical coherence tomography; CSFT, central subfield thickness; DRIL, disorganization of retinal inner layer; ERM, epiretinal membrane; ELM, external limiting membrane.

to anti-VEGF therapy (no visual improvement with persistent macular edema after at least 3 consecutive injections), alternative treatments were considered at the treating physicians' discretion. VA and CSFT were recorded at every visit; however, at months 12, 24 , and 36, primary analysis for proportion of eyes with VA and CSFT changes compared to baseline, number of intravitreal antiVEGF injections, and number of ophthalmologic clinic visits were evaluated.

\section{Statistical Analysis}

Demographics of patients were shown as mean (standard deviation, SD) or median (interquartile range, IQR) as appropriate for continuous data, while frequency (percentage) was used for categorical data. Snellen fraction VA was converted to approximate Early Treatment Diabetic Retinopathy Study (approxETDRS) letter scores and then used for statistical analysis [26]. Outcomes were analyzed first using actual observed data in patients presented at each 12-month interval time point (complete case analysis). Then, sensitivity analysis was performed for patients who were followed up through 12-, 24-, and 36-month visits. For missing VA and CSFT data, the last observation carried forward (LOCF) was imputed and calculated for primary analysis. To control for the correlated data of bilateral treatment and repeated measurements, multilevel analysis was performed. Mean change in VA and CSFT was analyzed by a generalized linear model, adjusted for gender, baseline VA, baseline CSFT, DR stage, number of injec-

Three-Year Outcomes of Anti-VEGF for

Diabetic Macular Edema tions, switching treatment group, presence of epiretinal membrane, presence of subretinal fluid, presence of intraretinal cyst, and disruption of ELM. DME duration and glycated HbAlc levels were not included in the analysis due to incomplete information. Proportion of eyes gaining or losing $\geq 10$ approxETDRS letters score was calculated. Statistical analysis was performed with STATA version 15 (StataCorp, College Station, TX, USA). A $p$ value $<0.05$ was considered statistically significant.

\section{Results}

\section{Study Population}

The 291 patients (430 eyes) who initiated the intravitreal anti-VEGF injection for diagnosis of DME between January 2012 and December 2016 and were followed-up for at least 12 months met eligibility criteria. Only 286 patients (423 eyes), for whom bevacizumab was the first anti-VEGF treatment, were evaluated in this study. Four eyes that initiated intravitreal anti-VEGF injection with ranibizumab and 3 eyes with aflibercept were excluded. The mean (SD) age was 56.8 (8.5) years. Overall, 146 of 286 (51.0\%) patients were female, and 137 of $286(47.9 \%)$ patients underwent 
Table 2. Number of injections and clinic visits during study period for the entire DME eyes stratified by baseline VA (complete case analysis)

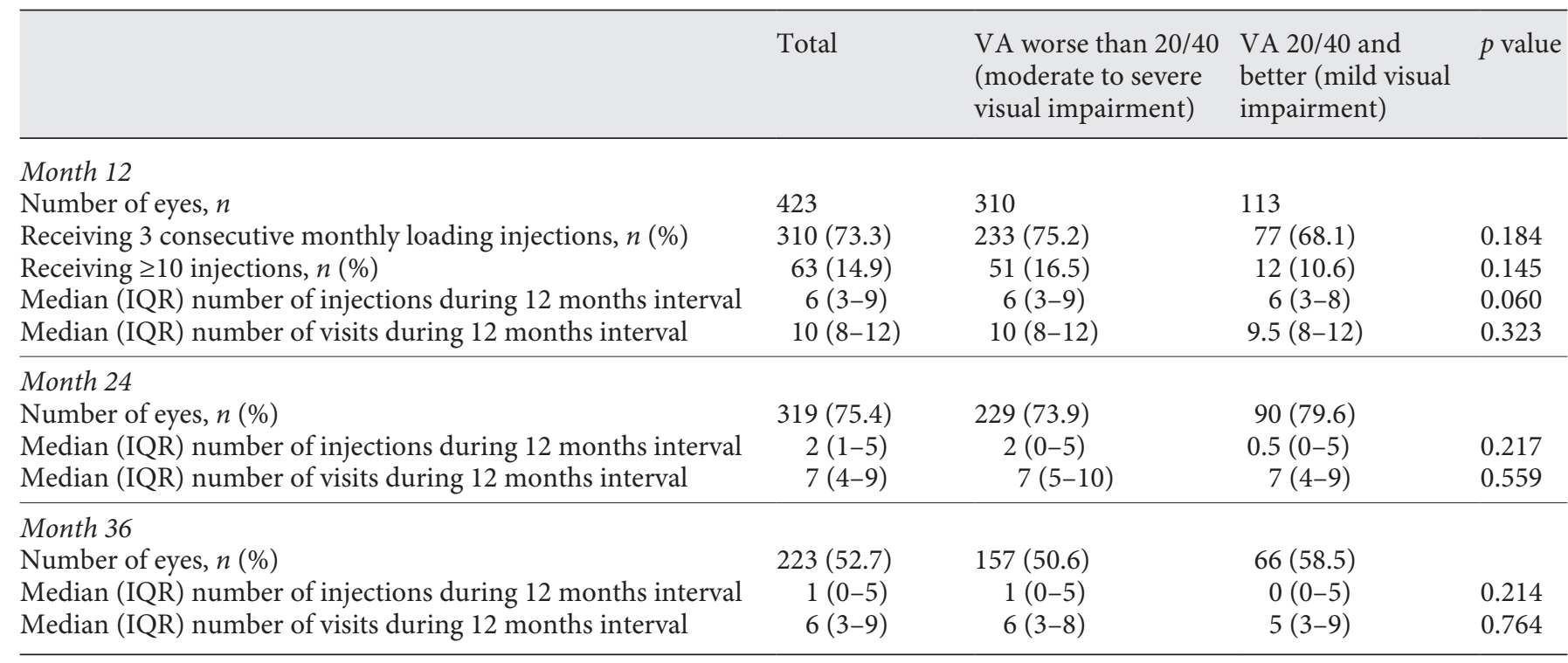

VA, visual acuity; IQR, interquartile range; DME, diabetic macular edema; SD, standard deviation.

bilateral eye treatment. Of 423 eyes, $319(75.4 \%)$ and 223 eyes $(52.7 \%)$ remained in clinic and had recorded data to assess up through 24 and 36 months, respectively. At baseline, a mean (SD) presenting VA of overall eyes was 50.2 (19.6) approxETDRS letters (20/100 Snellen equivalent), with 106 of 423 (25.1\%) eyes presenting VA of $\leq 35$ approxETDRS letters (20/200 Snellen equivalent). When the data were stratified according to baseline VA, the mild visual impairment group ( $\geq 70$ letters, $20 / 40$ and better Snellen equivalent) and the moderate to severe visual impairment group ( $<70$ letters, worse than 20/40 Snellen equivalent) had no significant differences in mean (SD) age (56.3 [7.7] vs. 57.1 [9.0], $p=0.375$ ) and proportions of female (55.3 vs. $49.5 \%, p=0.396)$. The presence of DRIL ( 64.2 vs. $46.0 \%$, $p<0.001$ ), disruption of ELM (38.4 vs. $7.1 \%, p<0.001)$, and epiretinal membrane ( 16.1 vs. $4.4 \%, p<0.001$ ) were shown at higher proportions in the moderate to severe visual impairment group than in the mild visual impairment group. Overall baseline demographics and ocular characteristics are summarized in Table 1.

\section{Frequency of Anti-VEGF Injections and Clinic Visits}

During the first 12 months, a median number of 6 (IQR 3-9) injections were administered. Among these, 310 eyes ( 310 of $423,73.3 \%$ ) received the 3 consecutive loading injections, while 90 eyes (90 of 423, 21.3\%) received $\geq 10$ injections. However, during the subsequent 12-month intervals, the reduction in median (IQR) number of injections to $2(1-5)$ and $1(0-5)$ was given in years 2 and 3 , respectively. The median (IQR) of clinic visits were 10 (8-12), 7 (4-9), and 6 (3-9) for the 12-month intervals of years 1, 2, and 3, respectively. Both baseline VA groups had similar numbers of intravitreal injections and clinic visits at 12, 24, and 36 months, respectively, as shown in Table 2.

\section{Visual Outcomes}

By complete case analysis, significant VA improvements compared to baseline were observed for the overall population at months 12,24 , and $36(+6.8,+4.1$, and +3.0 approxETDRS letter scores, $p$ values between 0.016 and $<0.001$ ), even though there was a slight decrease in trend of mean VA gain over the 36-month study period (as illustrated in Fig. 1a). When stratified by the baseline VA level, in the moderate to severe visual impairment group, significant VA gains $(+8.4,+6.9$, and +5.4 approxETDRS letter scores, all $p$ values $<0.001)$ were demonstrated; however, in the mild visual impairment group, the VA changes were not statistically significant $(+2.0,-3.5$, and -3.6 approxETDRS letter scores, $p$ value between 0.078 and 0.273 ) (Fig. 1b).

Details of mean changes in vision for the whole population and each baseline VA group are presented in Table 3 . However, by sensitivity analysis in mean VA chang- 
Table 3. Mean changes in VA compared to baseline for the entire DME eyes stratified by presenting VA groups (complete case analysis)

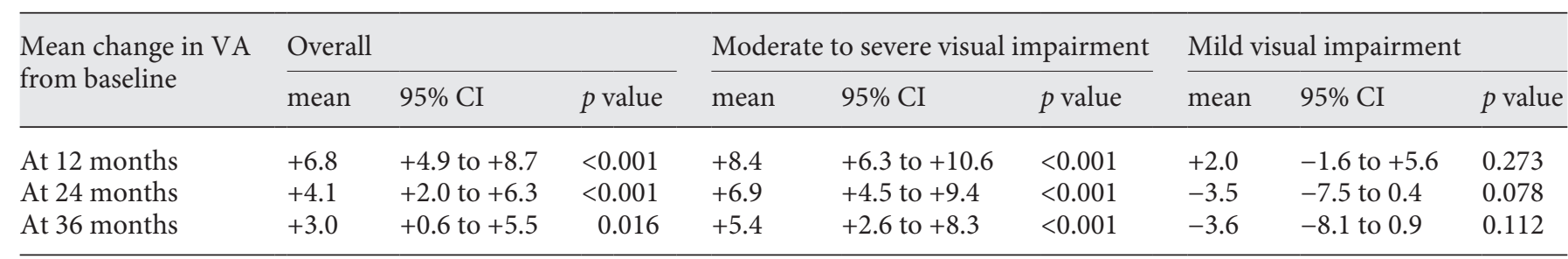

VA, visual acuity; CI, confidence interval; DME, diabetic macular edema.

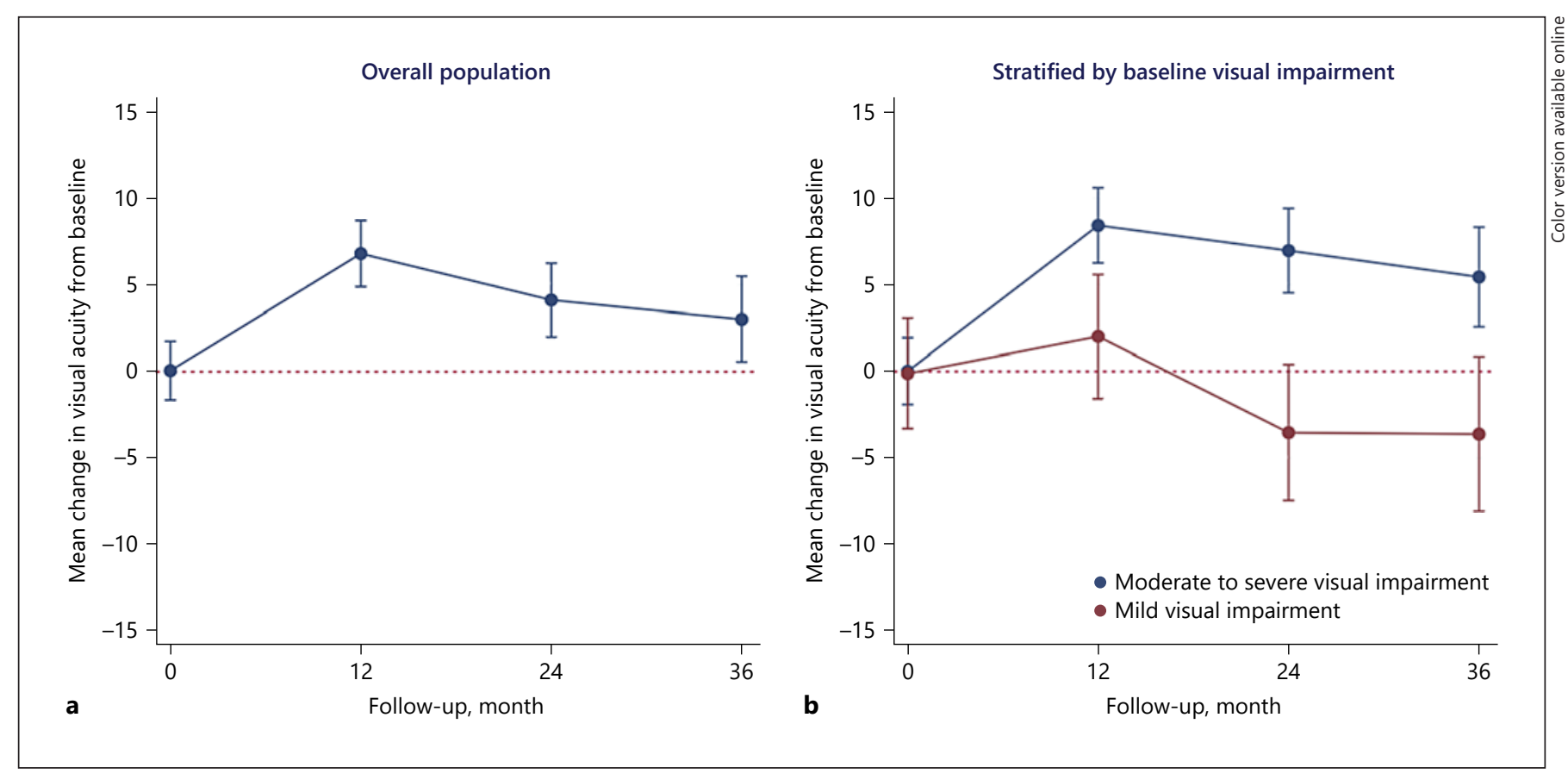

Fig. 1. Mean changes in VA from baseline over 36 months (complete case analysis). a Overall population. b Stratified by presenting VA groups. VA, visual acuity.

es by LOCF imputation, a comparable trend of changes between the moderate to severe visual impairment group $(+8.8,+8.0$, and +6.9 approxETDRS letter scores, all $p$ values $<0.001)$ and the mild visual impairment group $(+1.6$, -2.9 , and -3.3 approxETDRS letter scores, $p$ value between 0.065 and 0.325 ) is demonstrated in online suppl. Table 1 and online suppl. Figure 1 (for all online suppl. material, see www.karger.com/doi/10.1159/000512300).

\section{Anatomical Outcomes}

For the anatomical response, a significant reduction in CSFT, compared to the baseline, was demonstrated at month 12 and was maintained through months 24 and 36 (with a mean reduction of $-131.3,-161.0$, and $-162.3 \mu \mathrm{m}$, respectively, all $p$ values $<0.001)$ for the whole population. Complete case analysis (Table 4) and the LOCF model (online suppl. Table 2) showed a similarly significant decline in macular thickness in both the mild baseline visual impairment and moderate to severe visual impairment groups, all $p$ values $<0.001$. Figure 2 illustrates mean CSFT over time by complete case analysis, and online suppl. Figure 2 shows mean CSFT over time by LOCF analysis. By complete case analysis, the proportions (95\% confidence interval, CI) of eyes with persistent foveal thickening were $66.8 \%$ (61.0-72.5), 57.9\% (51.0-64.8), and $52.9 \%(44.8-60.9)$ at months 12,24 , and 36 , respectively, as demonstrated in online suppl. Table 3. Sensitivity analysis by LOCF showed similar trends of persistent 
Table 4. Mean changes in CSFT compared to baseline for the entire population and stratified by presenting VA groups (complete case analysis)

\begin{tabular}{|c|c|c|c|c|c|c|c|c|c|}
\hline \multirow{2}{*}{$\begin{array}{l}\text { Mean change } \\
\text { in CSFT from } \\
\text { baseline }\end{array}$} & \multicolumn{3}{|l|}{ Overall } & \multicolumn{3}{|c|}{ Moderate to severe visual impairment } & \multicolumn{3}{|c|}{ Mild visual impairment } \\
\hline & mean & $95 \% \mathrm{CI}$ & $p$ value & mean & $95 \% \mathrm{CI}$ & $p$ value & mean & $95 \% \mathrm{CI}$ & $p$ value \\
\hline At 12 months & -131.3 & -150.4 to -112.2 & $<0.001$ & -147.7 & -169.4 to -125.9 & $<0.001$ & -84.4 & -119.3 to -49.4 & $<0.001$ \\
\hline At 24 months & -161.0 & -182.6 to -139.5 & $<0.001$ & -188.2 & -213.4 to -163.1 & $<0.001$ & -92.2 & -130.0 to -54.4 & $<0.001$ \\
\hline At 36 months & -162.3 & -186.3 to -138.4 & $<0.001$ & -174.0 & -201.9 to -146.1 & $<0.001$ & -126.3 & -169.1 to -83.6 & $<0.001$ \\
\hline
\end{tabular}

CSFT, central subfield thickness; CI, confidence interval; VA, visual acuity.

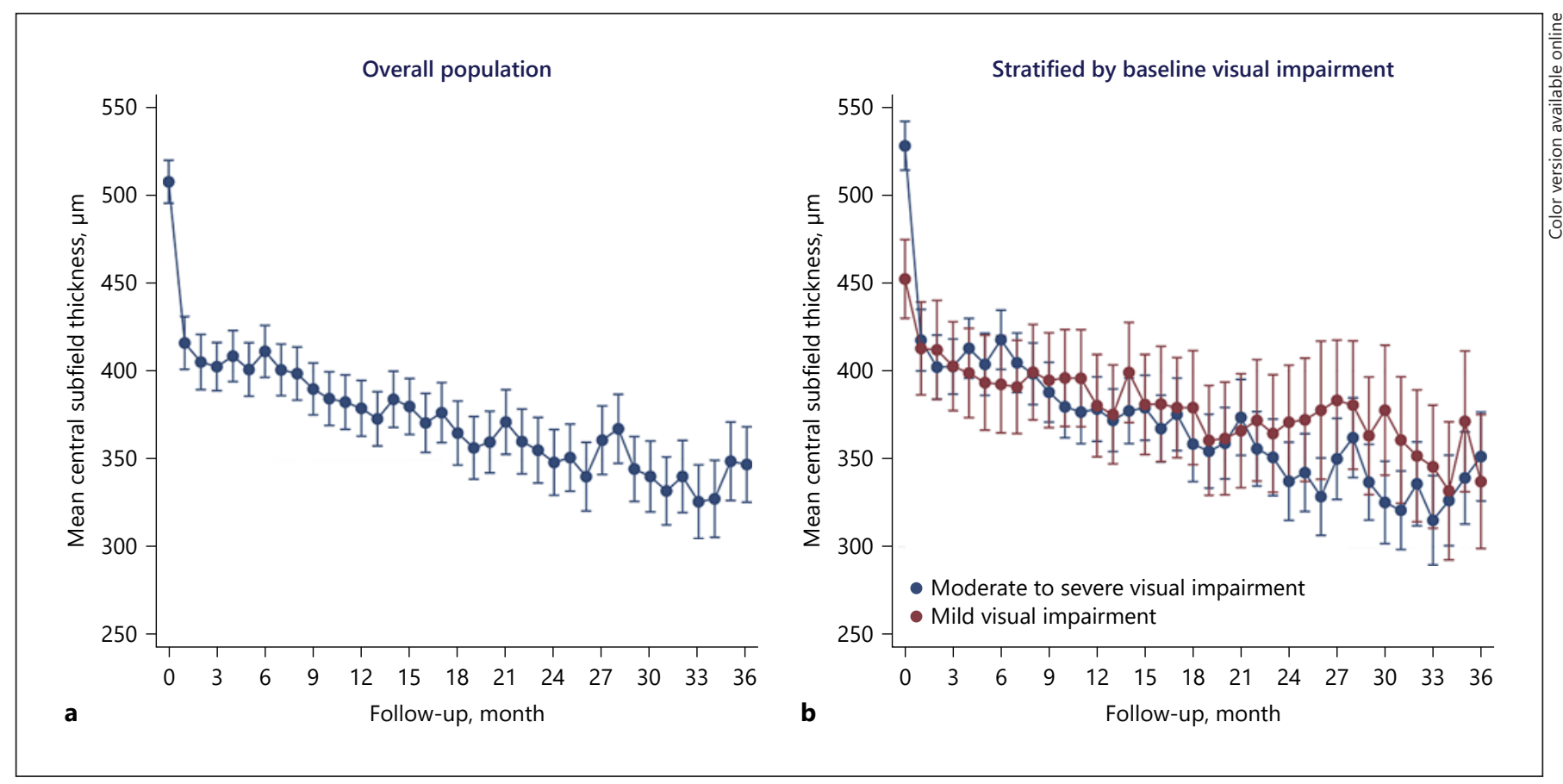

Fig. 2. a Mean CSFT over 36 months (complete case analysis). a Overall population. b Stratified by presenting VA groups. CSFT, central subfield thickness; VA, visual acuity.

macular edema at the similar time points (online suppl. Table 4). Moreover, in complete case analysis, the proportions of eyes $(95 \% \mathrm{CI})$ gaining $\geq 10$ approxETDRS letters at months 12,24 , and 36 were $43.1 \%$ (37.7-48.3), $38.4 \%$ (32.6-44.2), and $37.0 \%$ (30.1-43.9), whereas the proportions of eyes losing $\geq 10$ approxETDRS letters were $13.8 \%$ (9.9-17.6), 18.3\% (14.6-23.0), and 19.1\% (13.424.7), respectively (Fig. 3 ).

\section{Adjunctive Therapy}

During the study period, macular laser photocoagulation was performed in 182 of 423 eyes (43.0\%) and PRP in
170 of 423 eyes (40.2\%). Bevacizumab was the only intravitreal anti-VEGF administered during the entire study period in 308 of 423 (72.8\%) eyes. However, additional therapies were required in 115 of 423 eyes (27.2\%) at some time points including other anti-VEGF agents (ranibizumab and/or aflibercept) in 72 eyes (17\%), combined other antiVEGF agents and intravitreal dexamethasone implant (Ozurdex; Allergan, Irvine, CA, USA) in 18 eyes (4.3\%), other anti-VEGF agents followed by pars planar vitrectomy (PPV) in 2 eyes (0.5\%), intravitreal dexamethasone implant only in 9 eyes $(2.1 \%)$, intravitreal dexamethasone implant followed by PPV in 1 eye (0.2\%), and PPV only in 13 


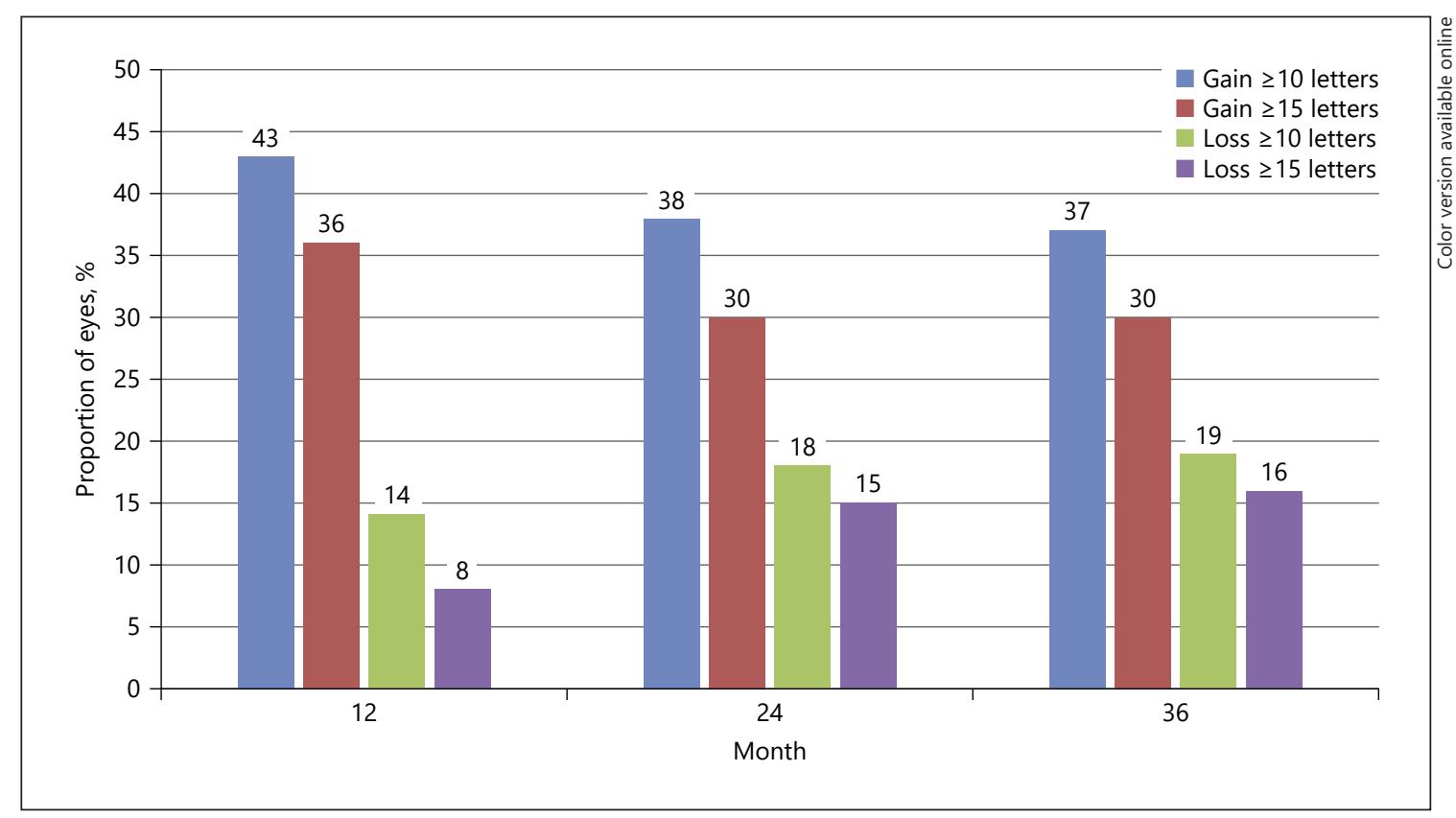

Fig. 3. Proportion of eyes gaining and losing VA compared to baseline for the entire population through the study period (complete case analysis). VA, visual acuity.

eyes (3.1\%). Excluding eyes that underwent vitrectomy, DME eyes that received combined bevacizumab and alternative intravitreal therapies, with a mean baseline VA of 51.1 (16.7) letters and a mean CSFT of $535.8(157.6) \mu \mathrm{m}$, experienced significant improvements in mean CSFT thickness by $-100.4 \mu \mathrm{m}$ (95\% CI: -147.2 to $-153.5, p<$ $0.001),-93.7 \mu \mathrm{m}(95 \% \mathrm{CI}:-140.0$ to $-46.6, p<0.001)$, and $-116.9 \mu \mathrm{m}$ (95\% CI: -165.4 to $-68.5, p<0.001)$ noted at months 12,24 , and 36 , respectively. However, there were nonsignificant improvements in mean VA at all 3 followup visits with +5.1 letters (95\% CI: -1.2 to $11.4, p=0.111$ ) at month $12,-0.2$ letters (95\% CI: -6.6 to $+6.1, p=0.946$ ) at month 24 , and +1.2 letters ( $95 \%$ CI: -5.3 to $+7.6, p=$ 0.719 ) at month 36 . Number of injections and clinic visits for eyes receiving combined intravitreal treatments are summarized in online suppl. Table 5.

Compared to the overall population, additional analysis for eyes receiving bevacizumab monotherapy (with no switching agent and/or underwent vitrectomy), adjusted for receiving PRP during treatments, exhibited similar responses of remarkable early visual gains during the first 12 months with a subsequent decline in the following 12-month intervals $(+7.1,+4.9$, and +2.5 letters at 12,24 , and 36 months, respectively [online suppl. Table 6]). In addition, similar trends of decreasing median (IQR) number of injections (5 [3-8], $0[0-3]$, and $0[0-2]$ at 12 ,
24 , and 36 months, respectively) are shown in online suppl. Table 7 , and the proportions of eyes gaining or losing vision through the study period are noted in online suppl. Table 8. In the subgroup analysis, the greater mean VA improvement at 12 months following treatments was observed in eyes receiving 3 initial monthly loading injections in both baseline VA groups (Fig. 4), while the benefits of $\geq 6$ injections associated with the higher VA gain only in the moderate to severe baseline visual impairment group as illustrated in online suppl. Figure 3.

\section{Discussion}

This study reflected a real-world visual response of intravitreal bevacizumab as initial treatment in CI-DME patients in a single clinical setting. For the whole study population, a mean VA improvement equivalent to 1 line (6.8 letters) from baseline was achieved with a median of 6 intravitreal anti-VEGF injections over the first 12 months. The amount of visual gain, though, was smaller than the eyes in RCT. Even with less frequent injections after the first 12-month period, with a median number of 2 between months 12 and 24 and 1 between months 24 and 36 , significant visual improvements were observed by mean of 4.1 and 3.0 letters, respectively. For the anatom- 
Fig. 4. Mean changes in VA at 12 months for DME eyes receiving intravitreal bevacizumab monotherapy stratified by presenting visual impairment and receiving of 3 loading injections. VA, visual acuity; DME, diabetic macular edema.

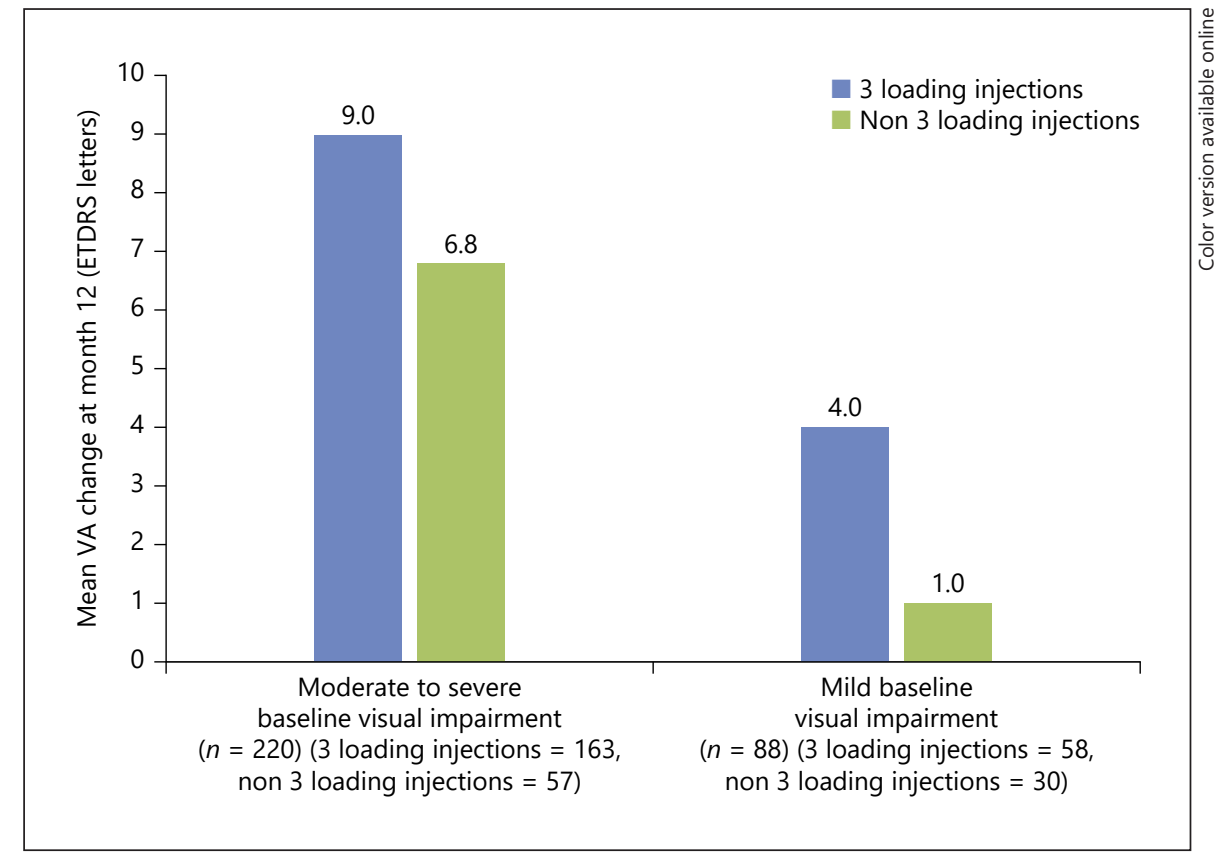

ical response, a significant CSFT reduction was recorded at the end of month 12 and throughout the follow-up period. Still, frequent ophthalmology clinic attendance (median numbers of 10, 7, and 6 visits in the first, second, and third 12-month periods, respectively) was required to attain these favorable outcomes.

In previous reports on real-world DME treatment, overall improvements in mean VA after intravitreal antiVEGF injections, regardless of agents, varied across publications $[19,21,27,28]$. In a prospective multicenter LUMINOUS study, DME eyes, treated with ranibizumab, exhibited country-specific visual improvements from -0.3 to 6.9 letters with a mean of $2.2-6.0$ injections at month 12 after the treatments [29]. In a 1-year Moorfields' real-world retrospective study, an improvement of 9.9 letters with an average of 6.9 aflibercept injections was reported [30]. The similar range of visual responses was also observed in our clinical setting. Nevertheless, when administering anti-VEGF treatment for DME, several associated factors that may influence the difference in visual changes between studies should be taken into consideration. Among these, presenting VA is one of the related parameters. A retrospective analysis of data from 9 clinical trials by Dugel et al. [31] has shown that in eyes with more severe visual loss, a greater visual improvement could be obtained, and vice versa. This relationship has also been supported in analysis of several real-world studies [19, 28, 31, 32]. Urbančič et al. [19] has revealed that in DME eyes with initial VA $\geq 70$ letters, the mean VA change was -3.9 letters at 2 years following anti-VEGF treatments while in eyes with initial VA $<70$ letters, the mean VA change was +5.7 letters. Biechl et al. [28] reported a similar trend at 5 years after starting anti-VEGF therapy; a mean VA improvement of 23 letters was noted in DME eyes with initial VA $\leq 35$ letters, whereas a mean VA change of -3 letters in eyes with initial VA $\geq 70$ letters.

Consistent with previous reports, the visual responses in our study were greatly impacted by presenting VA. A significant improvement in mean VA by 5.4 letters was observed in eyes with worse presenting VA ( $<70$ letters), compared to a nonsignificant VA loss of 3.6 letters in eyes with better presenting VA ( $\geq 70$ letters) at the end of the 36 -month period. This may partly be explained by the ceiling and floor effects of mean VA changes [21]. Notably, the presenting VA in this study (50 letters) was relatively low compared to other real-world publications (55-65 letters), which may partially attribute to a significant visual gain in this study $[16,17,27,28]$.

The heterogeneity of patients' characteristics including proportions of eyes with severe retinal ischemia with PDR, previous DME treatment, a longer duration of $\mathrm{DME}$, and delays in diagnosis and treatments may relate to variations in baseline VA between settings. The relatively high proportion of PDR in this study was likely associated with low levels of presenting VA. In public health perspectives, the increase in awareness of diabetic control 
and regular screening for DR/DME in diabetic populations should be emphasized.

The magnitude of visual responses and/or the ability to sustain visual improvement following anti-VEGF therapy also related to treatment frequency. With more frequent injections in clinical trials, higher visual gains were achieved than real-world reports $[11,13,15]$. This was supported by a recent real-world study that found the greater chance of VA improvement in the first year of treatment in eyes receiving either initial 3 monthly loading injections or overall $\geq 5$ injections [29]. Consistently, the correlation of injection frequency and visual outcome was also evidenced in studies from clinical practices [32,33]. Ciulla et al. [32] has shown in a retrospective review that a mean VA improvement had a linear relationship with the number of injections (from 3 or more injections) during the first year follow-up. Nevertheless, the need for frequent injections in the first year highlights a burden of this therapy for both patients and health-care providers that is difficult to adhere to in several settings. The possible explanations for the undertreatment $(<6$ injections in the first year) in our study could be difficulty in transportation to the health-care center, complexity of the referral systems, or other associated comorbidities that precluded both patients and physicians to schedule the more frequent treatments. However, this study confirms the benefits of receiving loading treatments and frequent injections in the first year of treatment. Therefore, this information should be presented to encourage the patients to have the treatment frequency as scheduled. Importantly, DME management options and/or regimens with less treatment frequency, but comparable efficacy, should be further investigated.

Apart from that mentioned above, the anti-VEGF agents used could be responsible for the variations in visual outcomes. In DRCR.net protocol T, significant visual gains had been reported among 3 anti-VEGF agents; however, the greater advantages of aflibercept, compared to bevacizumab, have been shown for DME eyes presenting with poor baseline VA [15]. Among real-world studies, a proportion of eyes that suffered a decline in vision of 2 or more lines was observed in $10-27 \%$ of the study populations $[16,17,19,20]$. This study noted that approximately one-fifth of the eyes lost 2 or more lines of vision during the observation period.

Chronicity of the macular edema may partially explain these outcomes. Several reports have shown that retinal microstructure features on OCT may reflect the chronicity and severity of retinal damage and were the significant prognostic factors of poor vision, even though these findings remain controversial [34-39]. In this study, more

Three-Year Outcomes of Anti-VEGF for Diabetic Macular Edema than half of the eyes presented with the DRIL and onethird with the disruption of ELM which could presumably impact visual responses following anti-VEGF treatments. However, differences in assessment algorithms (location, extent, and method of measurement) for these microstructural findings could attribute to the variation between studies. In this study, the retinal microstructures were assessed by manual segmentations of the OCT Bscan line passing through the fovea which may have underestimated some OCT biomarkers. In addition, the changes in OCT characteristics after treatment were not explored. Thus, the development of consensus guidelines or the computerized segmentation algorithms may improve an ability to determine the relationship of macular structure and vision responses.

A study in Taiwan demonstrated that a national healthcare policy is another factor related to visual improvements. With an increase in the reimbursement of intravitreal ranibizumab injections in Taiwan, the proportion of visual improvements increased [40]. Differences in reimbursement policy may be partly impacted with respect to different treatments and outcomes between countries.

Due to the retrospective nature of the review study, some systemic characteristic information including DM and DME duration, glycosylated HbA1c levels and associated diseases may be incomplete and limit the analyses. Furthermore, with no standardized VA measurement, an interpretation of vision has to be cautious. In addition, due to multiple treatment types in eyes undergoing switching or adding alternative therapies, efficacy of each therapeutic modality could not be accurately defined. However, this study provides additional longitudinal evidence for DME eyes that initiated treatment with bevacizumab in a generalized clinical setting.

\section{Conclusion}

This study provides relevant data for DME population who initiated treatment with anti-VEGF agents in realworld settings. Beneficial effects for reduction in macular thickening and improvement of vision during 3 years of follow-up were noted. Despite these promising outcomes, a decreasing trend of visual gains in the subsequent years warrants long-term regular monitoring. In addition, frequent treatment schedules and clinic visits remained one of the major obstacles to treatment. A therapy that could balance between efficacy and ophthalmologic visits while minimizing the burden of frequent injections warrants further investigation. 


\section{Statement of Ethics}

This study was conducted ethically in accordance with the World Medical Association Declaration of Helsinki. The study protocol was reviewed and approved by Research Ethics Committee, Faculty of Medicine, Chiang Mai University. Informed consent was waived due to anonymized data extraction without compromising the privacy of the subjects.

\section{Conflict of Interest Statement}

The authors have no conflicts of interest to declare.

\section{Funding Sources}

The authors did not receive any funding.

\section{Author Contributions}

J.C. designed the study, collected and analyzed the data, and wrote the draft paper. P.P. contributed to study methodology, analyzed the data, and proofed the final manuscript. A.T. contributed to study methodology, analyzed the data, and proofed the final manuscript. K.P. contributed to study methodology and proofed the final manuscript. P.K. contributed to study methodology and edited the manuscript. V.C. contributed to study methodology and edited the manuscript. N.W. contributed to study methodology and edited the manuscript.

\section{References}

1 Yau JW, Rogers SL, Kawasaki R, Lamoureux EL, Kowalski JW, Bek T, et al. Global prevalence and major risk factors of diabetic retinopathy. Diabetes Care. 2012 Mar;35(3):55664.

2 Ting DS, Cheung GC, Wong TY. Diabetic retinopathy: global prevalence, major risk factors, screening practices and public health challenges: a review. Clin Exp Ophthalmol. 2016 May;44(4):260-77.

3 Fenwick EK, Pesudovs K, Rees G, Dirani M, Kawasaki R, Wong TY, et al. The impact of diabetic retinopathy: understanding the patient's perspective. Br J Ophthalmol. 2011 Jun;95(6):774-82.

4 Man RE, Fenwick EK, Sabanayagam C, Li LJ, Tey CS, Soon HJ, et al. Differential impact of unilateral and bilateral classifications of diabetic retinopathy and diabetic macular edema on vision-related quality of life. Invest Ophthalmol Vis Sci. 2016 Sep;57(11):4655-60.

5 Fong DS, Fong DS, Strauber SF, Aiello LP, Beck RW, Callanan DG, et al. Comparison of the modified Early Treatment Diabetic Retinopathy Study and mild macular grid laser photocoagulation strategies for diabetic macular edema. Arch Ophthalmol. 2007 Apr; 125(4):469-80.

6 Beck RW, Beck RW, Edwards AR, Aiello LP, Bressler NM, Ferris F, et al. Three-year follow-up of a randomized trial comparing fo$\mathrm{cal} /$ grid photocoagulation and intravitreal triamcinolone for diabetic macular edema. Arch Ophthalmol. 2009 Mar;127(3):245-51.

7 Dugel PU, Bandello F, Loewenstein A. Dexamethasone intravitreal implant in the treatment of diabetic macular edema. Clin Ophthalmol. 2015;9:1321-35.

8 Photocoagulation for diabetic macular edema. Early Treatment Diabetic Retinopathy Study report number 1. Early Treatment Diabetic Retinopathy Study research group. Arch Ophthalmol. 1985 Dec;103(12):1796-806.
9 Elman MJ, Elman MJ, Aiello LP, Beck RW, Bressler NM, Bressler SB, et al. Randomized trial evaluating ranibizumab plus prompt or deferred laser or triamcinolone plus prompt laser for diabetic macular edema. Ophthalmology. 2010 Jun;117(6):1064-e35.

10 Nguyen QD, Shah SM, Khwaja AA, Channa R, Hatef E, Do DV, et al. Two-year outcomes of the ranibizumab for edema of the macula in diabetes (READ-2) study. Ophthalmology. 2010 Nov;117(11):2146-51.

11 Nguyen QD, Brown DM, Marcus DM, Boyer DS, Patel S, Feiner L, et al. Ranibizumab for diabetic macular edema: results from 2 phase III randomized trials: RISE and RIDE. Ophthalmology. 2012 Apr;119(4):789-801.

12 Rajendram R, Fraser-Bell S, Kaines A, Michaelides M, Hamilton RD, Esposti SD, et al. A 2-year prospective randomized controlled trial of intravitreal bevacizumab or laser therapy (BOLT) in the management of diabetic macular edema: 24-month data: report 3. Arch Ophthalmol. 2012 Aug;130(8):972-9.

13 Korobelnik JF, Do DV, Schmidt-Erfurth U, Boyer DS, Holz FG, Heier JS, et al. Intravitreal aflibercept for diabetic macular edema. Ophthalmology. 2014 Nov;121(11):2247-54.

14 Schmidt-Erfurth U, Lang GE, Holz FG, Schlingemann RO, Lanzetta P, Massin P, et al. Three-year outcomes of individualized ranibizumab treatment in patients with diabetic macular edema: the RESTORE extension study. Ophthalmology. 2014 May;121(5): 1045-53.

15 Wells JA, Glassman AR, Ayala AR, Jampol LM, Bressler NM, Bressler SB, et al. Aflibercept, Bevacizumab, or Ranibizumab for diabetic macular edema: two-year results from a comparative effectiveness randomized clinical trial. Ophthalmology. 2016 Jun;123(6): 1351-9.
16 Patrao NV, Antao S, Egan C, Omar A, Hamilton R, Hykin PG, et al. Real-world outcomes of ranibizumab treatment for diabetic macular edema in a United Kingdom national health service setting. Am J Ophthalmol. 2016 Dec;172:51-7.

17 Holekamp NM, Campbell J, Almony A, Ingraham $\mathrm{H}$, Marks S, Chandwani $\mathrm{H}$, et al. Vision outcomes following anti-vascular endothelial growth factor treatment of diabetic macular edema in clinical practice. Am J Ophthalmol. 2018 Jul;191:83-91.

18 Ziemssen F, Wachtlin J, Kuehlewein L, Gamulescu MA, Bertelmann T, Feucht N, et al. Intravitreal ranibizumab therapy for diabetic macular edema in routine practice: two-year real-life data from a non-interventional, multicenter study in Germany. Diabetes Ther. 2018 Dec;9(6):2271-89.

19 Urbančič M, Klobučar P, Zupan M, Urbančič K, Lavrič A. Anti-VEGF treatment of diabetic macular edema: two-year visual outcomes in routine clinical practice. J Ophthalmol. 2020; 2020:6979758.

20 Arevalo JF, Lasave AF, Wu L, Acon D, Farah ME, Gallego-Pinazo R, et al. Intravitreal bevacizumab for diabetic macular oedema: 5-year results of the Pan-American Collaborative Retina Study group. Br J Ophthalmol. 2016 Dec;100(12):1605-10.

21 Ciulla TA, Bracha P, Pollack J, Williams DF. Real-world outcomes of anti-vascular endothelial growth factor therapy in diabetic macular edema in the United States. Ophthalmol Retina. 2018 Dec;2(12):1179-87.

22 Farinha C, Martins A, Neves A, Soares R, Ruão $M$, Ornelas $M$, et al. Ranibizumab for the treatment of diabetic macular oedema in the real-world clinical setting in Portugal: a Multicentre Study. Ophthalmologica. 2019; 241(1):1-8. 
23 Park KH, Kim YY, Jo YJ, Oh J, Lee JE, Lee JE, et al. Healthcare utilization and treatment patterns in diabetic macular edema in korea: a retrospective chart review. J Korean Med Sci. 2019 Apr;34(15):e118.

24 Korobelnik JF, Daien V, Faure C, Tadayoni R, Giocanti-Auregan A, Dot C, et al. Real-world outcomes following 12 months of intravitreal aflibercept monotherapy in patients with diabetic macular edema in France: results from the APOLLON study. Graefes Arch Clin Exp Ophthalmol. 2020 Mar;258(3):521-8.

25 Chalam KV, Bressler SB, Edwards AR, Berger BB, Bressler NM, Glassman AR, et al. Retinal thickness in people with diabetes and minimal or no diabetic retinopathy: Heidelberg Spectralis optical coherence tomography. Invest Ophthalmol Vis Sci. 2012 Dec;53(13): 8154-61.

26 Gregori NZ, Feuer W, Rosenfeld PJ. Novel method for analyzing snellen visual acuity measurements. Retina. 2010 Jul-Aug;30(7): 1046-50.

27 Maggio E, Sartore M, Attanasio M, Maraone G, Guerriero M, Polito A, et al. Anti-vascular endothelial growth factor treatment for diabetic macular edema in a real-world clinical setting. Am J Ophthalmol. 2018 Nov; 195: 209-22.

28 Biechl AC, Bhandari S, Nguyen V, Arnold JJ, Young S, Fraser-Bell S, et al. Changes in realworld treatment patterns for diabetic macular oedema from 2009-2019 and five-year outcomes: data from the Fight Retinal Blindness! Registry. Clin Exp Ophthalmol. 2020 Aug; 48(11):802-12.
29 Mitchell P, Sheidow TG, Farah ME, Mahmood S, Minnella AM, Eter N, et al. Effectiveness and safety of ranibizumab $0.5 \mathrm{mg}$ in treatment-naïve patients with diabetic macular edema: Results from the real-world global LUMINOUS study. PLoS One. 2020;15(6): e0233595.

30 Lukic M, Williams G, Shalchi Z, Patel PJ, Hykin PG, Hamilton RD, et al. Intravitreal aflibercept for diabetic macular oedema in real-world: 36-month visual acuity and anatomical outcomes. Eur J Ophthalmol. 2020 May:1120672120925034.

31 Dugel PU, Hillenkamp J, Sivaprasad S, Vögeler J, Mousseau MC, Wenzel A, et al. Baseline visual acuity strongly predicts visual acuity gain in patients with diabetic macular edema following anti-vascular endothelial growth factor treatment across trials. Clin Ophthalmol. 2016;10:1103-10.

32 Ciulla TA, Pollack JS, Williams DF. Visual acuity outcomes and anti-VEGF therapy intensity in diabetic macular oedema: a realworld analysis of 28658 patient eyes. $\mathrm{Br} \mathrm{J}$ Ophthalmol. 2020 Apr 7:

33 Cantrell RA, Lum F, Chia Y, Morse LS, Rich WL, Salman CA, et al. Treatment patterns for diabetic macular edema: an intelligent research in Sight (IRIS ${ }^{\circ}$ ) Registry Analysis. Ophthalmology. 2020 Mar;127(3):427-9.

34 Kim M, Lee P, Kim Y, Yu SY, Kwak HW. Effect of intravitreal bevacizumab based on optical coherence tomography patterns of diabetic macular edema. Ophthalmologica. 2011;226(3):138-44.
35 Sun JK, Lin MM, Lammer J, Prager S, Sarang $\mathrm{R}$, Silva PS, et al. Disorganization of the retinal inner layers as a predictor of visual acuity in eyes with center-involved diabetic macular edema. JAMA Ophthalmol. 2014 Nov; 132(11):1309-16.

36 Seo KH, Yu SY, Kim M, Kwak HW. Visual and morphologic outcomes of intravitreal ranibizumab for diabetic macular edema based on optical coherence tomography patterns. Retina. 2016 Mar;36(3):588-95.

37 Gerendas BS, Bogunovic H, Sadeghipour A, Schlegl T, Langs G, Waldstein SM, et al. Computational image analysis for prognosis determination in DME. Vision Res. 2017 10;139: 204-10.

38 Fickweiler W, Schauwvlieghe AME, Schlingemann RO, Maria Hooymans JM, Los LI, Verbraak FD, et al. Predictive value of optical coherence tomographic features in the bevacizumab and ranibizumab in patients with diabetic macular edema (brdme) study. Retina. 2018 Apr;38(4):812-9.

39 Santos AR, Costa MÂ, Schwartz C, Alves D, Figueira J, Silva R, et al. Optical coherence tomography baseline predictors for initial bestcorrected visual acuity response to intravitreal anti-vascular endothelial growth factor treatment in eyes with diabetic macular edema: the CHARTRES Study. Retina. 2018 Jun; 38(6):1110-9.

40 Lai TT, Hsieh YT, Yang CM, Ho TC, Yang $\mathrm{CH}$. Effect of reimbursement policy on visual outcomes in patients with diabetic macular edema treated with ranibizumab. Retina. 2020 Nov;40(11):2019-97. 